The binding screw (A) being free (or loose), the driving disc (B) is disengaged from the polar axis. On turning the winding-up wheel (C) the plunger (D) is drawn out of its cylinder (E), sucking oil into the cylinder from the reservoir (F) through a valve. The winding-up has also turned the driving disc (B) and lifted the driving weight $(G)$. When the winding-up is finished, the cylinder (E) is full of oil, which is imprisoned by the automatic closing of the valve from the reservoir (F). The oil is now under pressure, caused by the driving weight $(G)$, and it can only get out of the cylinder (E) back to the reservoir, through a hole, the size of which is controlled by the regulating screw $(\mathrm{H})$. If the binding screw (A) is now screwed fast, the telescope will turn on its polar axis at the rate allowed by the regulating screw $(\mathrm{H})$.

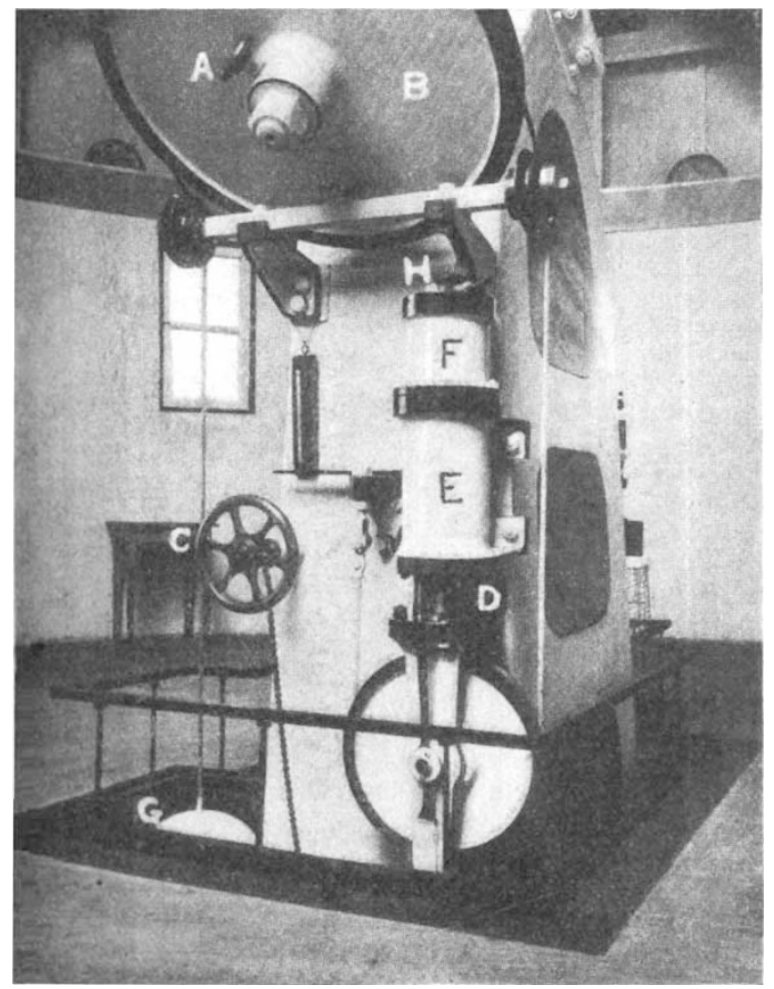

An oil possessing a high flash point is suitable for this purpose, since the change in viscosity under different temperatures is then not much felt. A change in the viscosity causes the oil to pass through the valve at a correspondingly different rate. Hence it is necessary to adjust the valve to the local temperature, which is done by setting the regulating screw according to the positions representing degrees of temperature, which are marked round the top of the oil reservoir. The regulator runs for three hours before re-winding, the oil, of course, being used over and over again.

In a mechanism of this nature it is evident that the question of friction is of the first importance, and great care has been taken, first, to reduce the working friction to a minimum, and, secondly, to make the unavoidable friction as constant as possible. The polar axis is therefore fitted with three sets of ball bearings (two axial and one thrust); the guide pulleys are also fitted with ball bearings, and the plunger is ground truly parallel and very smooth.

The regulator is intended more especially for eclipse and visual work in general. There are slight errors of NO. 2446 , VOL. 98$]$ motion which have yet to be overcome, and they render it difficult to keep a star on the wire; but by an additional contrivance which has yet to be adapted the errors in question might be reduced sufficiently to render the device suitable for stellar photography.

The Observatory, Bramley, Yorkshire, Scriven Bolton.

$$
\text { August } 23 \text {. }
$$

\section{On the "Wolf-note" of the Violin and 'Cello.}

IN a letter to Nature (June 29) on the wolf-note of the violin and 'cello which has recently come to our notice, Mr. Raman referred to a paper by one of us (G. W. W.) on the subject. We had anticipated being associated in a joint paper dealing more fully with the results when the war put an end to the work.

At the time when the preliminary results were published the cyclical variation in intensity of the wolf-note was provisionally attributed to the beating of the oscillations of the two tuned resonators, belly and string, "coupled" together by the bridge, and not to transitory beats, as perhaps justly inferred by $\mathrm{Mr}$. Raman from the wording of the paper, it being assumed that the strong reaction of the belly on the string interfered with even bowing. But the later results we obtained could not be reconciled with such a view, and we were seeking an explanation on the lines of that now given by Mr. Raman.

Thus further records of the wolf-note showed very clearly indeed the prominience of the octave at the minima in the belly records. Also simultaneous records of belly and string vibrations led, in the case of one 'cello, to curves practically identical with those given by Mr. Raman, in which the maxima and minima of belly amplitude lag behind those of the string by a quarter of a cycle. With another 'cello of brilliant quality no appreciable lag appeared to exist, though perhaps better photographs would have shown some. Finally, the frequency in variation of intensity seemed to depend on the speed of the bow and not on the form of the bridge, which would affect the degree of coupling.

We take this opportunity of recording two other results obtained by us, which furnish still more evidence for the belief that the wolf-note is a phenomenon accompanying maximum resonance. They are worthy of record, because this interpretation of the effect is not that which is held generally by musicians and instrument-makers.

The first was obtained in some experiments on "the mute," With the two 'cellos with which observations were made, the effect of loading the bridge with the mute was a reduction in pitch of the wolf, by an amount depending on the form of the mute. Thus, with one heavy mute the pitch was lowered from $G$ to $D$, and with a lighter mute from $G$ to $E$. By blowing a cornet in front of the belly, it was found that the natural frequency of the belly was correspondingly lowered.

The second was obtained when vibrations of the belly were set up by plucking a string. The transitory beats present in the early stages of a forced oscillation were very clearly shown. The $G$ string was plucked to give a note within a few semitones of the wolf-note. After six or seven beats the belly took up the sinusoidal vibration of the string, which persisted until the sound was no longer audible. Calculations of the natural period of the belly from the frequency of the beats led in each case to a period the same as that of the wolf-note and that given by the cornet.

A. M. Tyñdalt. G. W. White.

University of Bristol, September I. 